\title{
Rapid Iron-On User Interfaces: Hands-on Fabrication of Interactive Textile Prototypes
}

\author{
Konstantin Klamka ${ }^{1}$, Raimund Dachselt ${ }^{1,2}$, Jürgen Steimle ${ }^{3}$ \\ ${ }^{1}$ Interactive Media Lab Dresden, Technische Universität Dresden, Germany \\ ${ }^{2}$ Centre for Tactile Internet with Human-in-the-Loop (CeTI), Technische Universität Dresden, Germany \\ ${ }^{3}$ Saarland University, Saarland Informatics Campus, Saarbrücken, Germany \\ klamka@acm.org,dachselt@acm.org, steimle@cs.uni-saarland.de
}
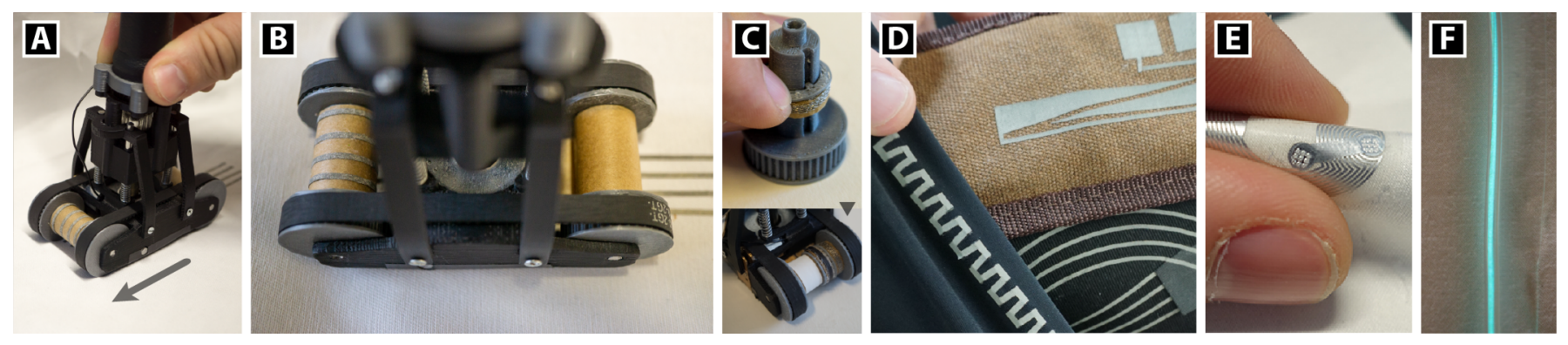

Figure 1. Rapid Iron-On User Interfaces constitute a new approach for fabrication of custom textile prototypes in a fast and intuitive sketching-like manner. By using a handheld ironing tool $(A+B)$ for applying adhesive functional tapes and patches $(C-E)$, the designer can create rich interactive functionality, drawing from a library of thin and flexible wiring, input and output (D-F) as well as computing components.

\begin{abstract}
Rapid prototyping of interactive textiles is still challenging, since manual skills, several processing steps, and expert knowledge are involved. We present Rapid Iron-On User Interfaces, a novel fabrication approach for empowering designers and makers to enhance fabrics with interactive functionalities. It builds on heat-activated adhesive materials consisting of smart textiles and printed electronics, which can be flexibly ironed onto the fabric to create custom interface functionality. To support rapid fabrication in a sketching-like fashion, we developed a handheld dispenser tool for directly applying continuous functional tapes of desired length as well as discrete patches. We introduce versatile compositions techniques that allow for creating complex circuits, utilizing commodity textile accessories and sketching custom-shaped I/O modules. We further contribute a comprehensive library of components for input, output, wiring and computing. Three example applications, results from technical experiments and expert reviews demonstrate the functionality, versatility and potential of this approach.
\end{abstract}

\section{Author Keywords}

E-Textile; Wearable UI; Fabrication; Prototyping; Iron-on.

\section{CCS Concepts}

-Human-centered computing $\rightarrow$ Interaction devices; Interaction techniques; User interface toolkits;

Permission to make digital or hard copies of part or all of this work for personal or classroom use is granted without fee provided that copies are not made or distributed for profit or commercial advantage and that copies bear this notice and the full citation on the first page. Copyrights for third-party components of this work must be honored For all other uses, contact the Owner/Author(s).

CHI '20, April 25-30, 2020, Honolulu, HI, USA.

(C) 2020 Copyright is held by the owner/author(s).

ACM ISBN 978-1-4503-6708-0/20/04.

http://dx.doi.org/10.1145/3313831.3376220

\section{INTRODUCTION}

Recent advances in functional textiles and electronics miniaturization are bringing a new generation of wearable user interfaces that are deeply integrated into clothing and other objects made of fabric. Their ergonomics, softness, direct reachability and close body contact, alongside other benefits, make them an outstanding platform for mobile computing and interaction. As current research demonstrates, smart textiles and functional yarns can already have multi-functional properties and offer a rich set of sensing and output capabilities, for instance to dynamically adapt their appearance [36, 16], light up [39] or heat [3], store information [9], track and micromanage body movements [13] or even harvest its own energy by using motion or solar generators [10].

While these textile innovations are suggesting that wearable computing devices may hit the market in a not-so-distant future, it remains an open question how to support designers and technology-enthusiastic makers with fabrication tools to create, iterate and evaluate functional prototypes at an early design stage. Unfortunately, the fabrication of textile sensors and traces on the yarn-level, like sewing, weaving, knitting, or embroidering, is commonly quite time-consuming and cumbersome when done manually. Automating these processes requires expert machinery and knowledge typically outside the realm of a prototyping space. While we do not lay the focus on creating industrial strength prototypes, our goal is to accelerate rapid prototyping of smart textiles and to considerably simplify the design process for hobbyists, makers, interaction and textile designers, including both novices and advanced users, particularly in the early design stage.

Therefore, we present Rapid Iron-On (RIO) User Interfaces, a novel approach for easy fabrication of custom interactive 
textile prototypes in a sketching-like fashion. Inspired by the simplicity and versatility of package and sewing tapes as well as textile stickers and patches, our approach is based on functional textile adhesive tapes and patches that each implement a specific functionality. We contribute a handheld general-purpose ironing tool that combines the dispensing and bonding of various functional materials into a single, rapid and consistent process. Our key innovation comprises an additive fabrication concept consisting of this novel device as well as tapes and patches that in combination allow to physically sketch and compose functional electrical circuits and versatile interface functionalities directly onto textiles.

We start by presenting the concept of sketching with functional textile materials and the handheld ironing tool. Continuous tapes can be applied in custom length and shape, to create circuitry and other line-type elements, while discrete patches can be used to create components. Flexible composition techniques allow the designer to arrange and layer these elements to create functional circuits, sketch pre-defined or customshaped I/O modules, and integrate textile accessories.

We further demonstrate that the iron-on approach is compatible with a wide array of components made of textile functional materials, printable electronics, or with embedded flexible PCBs. We contribute a comprehensive library of iron-on components that function as input (e.g., touch, bend, shear force, NFC identification), output (e.g., electro-luminescent, LED and eink display, haptic and vibrotactile output), wiring (e.g., bus lines, shielded conductors), energy supply (e.g., solar power harvesting) and processing (e.g., flexible micro controllers).

We validate our approach in a series of technical studies, expert interviews, and implemented example applications. The results demonstrate the utility and versatility of our approach, confirm its ease and rapidity and show that it enables prototyping wearable computing applications that offer rich interactive functionality.

In summary, the main contributions of this paper are:

- A fabrication technique for rapid fabrication of functional e-textiles that allows to physically sketch and compose a variety of rolled-up, functional materials and modular patches onto textiles.

- The realization of a handheld ironing tool that enables creating complex circuits, sketching pre-defined or customshaped I/ O modules and integrating textile accessories.

- A comprehensive library of components consisting of functional tapes and patches for input, output, wiring, energy supply and processing.

- Three implemented example applications that demonstrate how our approach can be conveniently used for the fast and easy prototyping of wearable computing applications.

- Results from technical experiments and three expert reviews confirm the technical functionality and investigate design strategies and usage potential of the approach.

\section{RELATED WORK}

Our work is related to toolkits and fabrication techniques for e-textiles and to research on interactive personal fabrication.

\section{Toolkits for E-Textiles \& Fabrication Techniques}

Previous research on electronic textiles has investigated several functional materials including conductive, resistive and piezoresistive yarns, coatings or fabrics to integrate electrical circuits and I/O modules including stretch [63], touch [47, 55], and pressure [49, 48, 22, 62] input. Depending on the specific material and intended purpose, different yarn-based or additive manufacturing processes are required.

Sewing, weaving [4], knitting [24] or embroidering [54] methods aim to incorporate digital functionalities directly into textiles by using functional yarns and threads that seamlessly merge with the fabric or even create a smart fabric itself. While the results have a high degree of integration (cf. [55]), machines used in textile industries are typically inaccessible for designers and makers and often incompatible with low volume prototyping. They also require specialized skills, knowledge and production time.

In order to make textile prototyping accessible for a broader audience, some research has been conducted to either contribute new fabrication techniques for e-textile prototyping or provide wearable construction toolkits. For example, Buechley et al. [7, 5] introduced LilyPad that allows to easily connect electronic components with conductive yarns. In addition, construction toolkits like i*CATch [43], EduWear [35], MakerWear [37], Schnittmuster [41], or BodyHub [50] empower users to create their own e-textile prototypes by using I/O modules. Finally, computer-aided knitting and design tools like AdaCAD [26], KnitPicking Textures [31] or Knitting Skeletons [34] have been introduced to reduce design complexity.

As an alternative to e-sewing, Buechley et al. [6] introduced fabric PCBs consisting of laser-cut conductive fabric and ironon adhesive to integrate traditional electronics and novel textile sensors. We have taken inspiration from this early work that laid the foundations for ironing conductive fabrics onto garments. In this line, Perner-Wilson et al. [53] further explore electrical properties of materials by using craft techniques to build electronics that emphasize materiality and aesthetics. In addition, research on interactive electronic tapes, like ShapeTape [29], TouchTape [71] or SensorTape [14], demonstrate how tapes can be utilized to enhance everyday objects.

\section{Interactive Personal Fabrication}

Digital fabrication processes are commonly characterized by sequential workflows where engineers first create a design and afterwards send their design to a digital fabrication machine. In contrast, interactive fabrication focuses on real-time control already during the fabrication process and aims to closely link the designer's input and the machine's physical output (cf. [70]). Therefore, several digital fabrication machines like laser cutters [42], CNC routers [40], thermoforming machines [40], 3D printers [51], special fabric 3D printers for interactive soft objects [52] as well as embroidery machines [30] have been investigated. Furthermore, ad-hoc customization of printed electronic components has been discussed for cuttable multi-touch sensors [45], screen printed electroluminescence patches [46] and multi-material inkjet printing approaches [38] that are capable of directly printing on iron-on material. In addition, LASEC [28] allows to rapidly 
laser cut circuits with custom stretchability. Closely related to our work is also Sketch\&Stitch [30], a system that allows a designer to first sketch the required design with pens onto a textile while in a second fabrication step a computationally controlled embroidery machine realizes the design with functional yarns. Our work shares the idea of directly designing and working on the textile itself. We aim to combine design and fabrication into a single process by using an additive ironing of functional materials.

\section{THE RAPID IRON-ON CONCEPT}

With Rapid Iron-On (RIO) we present a new prototyping principle for e-textiles that allows to physically "sketch" user interfaces in a hands-on manner directly onto textiles. Our approach builds on functional textile patches, in continuous tapes or discrete patches, which comprise heat-activated adhesive to be easily ironed onto the base textile. Those patches are made of smart fabrics and printed thin-film technologies, offering a rich variety of electronic functions while preserving soft and flexible properties. A new handheld iron tool allows the designer to rapidly add functional patches, similarly to making a sketch with a pen. For instance, she can create conductive traces with desired properties by "drawing" lines (Figure 5), and can add functional patches for specific I/O functionality (Figure 3), circuit design, power supply, etc. By flexibly combining, layering and juxtaposing, she can create custom interfaces with rich functionality. For difficult-to-reach regions, ironing with a small-scale iron is also supported.

\section{Sketching with Functional Textile Materials}

The idea of sketching functional textile patches on a base fabric implies some key requirements the approach had to fulfill. In most cases, patches should match the visio-haptic appearance of the base material, should be thin, stretchable, robust, and durably bonded with the textile. In addition to these properties, textile patches should be available in various form factors, whether short or long, narrow or wide, curved or straight. For example, for routing traces that are part of a textile circuit, it is crucial to support continuous lines of customizable length and shape. In addition to conductors, other functional elements also belong to the continuous-line type: linear input elements, such as sliders or bend sensors, or elements that light or heat up. In contrast, most other components of a circuit rather exhibit a fixed shape, mostly well-defined in advance. Examples are touch or pressure buttons, displays, or matrix sensors. They need to be freely aligned and connected to conductors.

We therefore propose two types of textile patches that can be freely arranged on the fabric and simply fixed by using our handheld RIO tool: continuous tapes and discrete patches.

Continuous RIO Tapes are functional textile patches that are rolled on spools and can be ironed-on in a seamless fashion (see Figure 5) in custom-defined lengths and variable shapes. Designers have the choice of various functional tapes including simple materials (e.g., conductive fabric, see Figure 4) and prefabricated component tapes (e.g., shielding or pressuresensitive properties).

Functional RIO Patches are pre-defined in their shape and do not have the continuous, rollable form factor of tapes. They

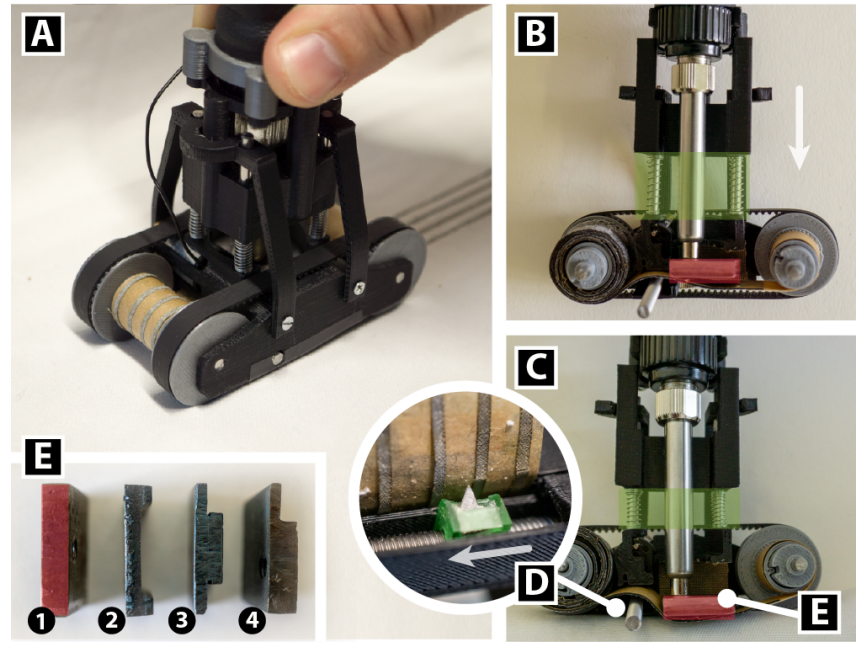

Figure 2. Iron-On working principle: A functional textile component is fabricated using the dispenser (A). When not pressed down, the tape is slightly lifted above the surface (B). When pressed down by the exchangeable, spring-loaded heating block $(E)$, the tape is ironed onto the textile $(\mathrm{C}+\mathrm{A})$. The cutting blade (D) moves along an actuated leadscrew.

also seamlessly fit into our process of rapid ironing since they provide compatible properties (e.g., connector pitches). A RIO patch can be active or passive. Active patches enable different levels of I / O capabilities, while passive patches serve as construction material for, e.g., routing or isolating traces.

The synergistic interplay of sketching with continuous tapes and composing with functional patches (see Figure 3) facilitates a seamless and versatile workflow, avoiding major interruptions that would be caused by changing between tools.

\section{The Rapid Iron-On Tool}

In principle, any household iron could be used to ironing-on tapes and patches. However, it would lack the ease, directness, and precision of sketching. In order to allow designers to apply functional tapes and patches in a rapid and controllable manner, we contribute the RIO tool as a handheld device that is capable of directly dispensing and iron-bonding materials at the same time. Due to its slim form factor, it allows to dispense continuous functional material rolled on spools directly onto the fabric, similar to the way one sketches with a pen. The tool also allows to quickly bond discrete patches onto the material with high precision.

Technically, our prototype (Figure 2, A-E) is based on a springloaded heating block that temporarily pushes $(\mathrm{B}+\mathrm{C})$ an iron-on tape onto the textile and thereby transfers the patch using heat activation. This resembles the idea of pressing down a marker pen to draw a line. To achieve a slim form factor and support precise "sketching", we use a modified solder iron with a screwable tip that allows to change heating elements (E) and to adjust heating power when required for delicate materials.

Continuous tapes are rolled onto spools, which can be easily inserted into the RIO tool and exchanged when needed. We built the RIO tool with two wheel axles such that it can hold spools (see Figure 2, A). An empty spool on the opposite side takes the end of the tape (see cross-cut in Figure 2, B). This resem- 

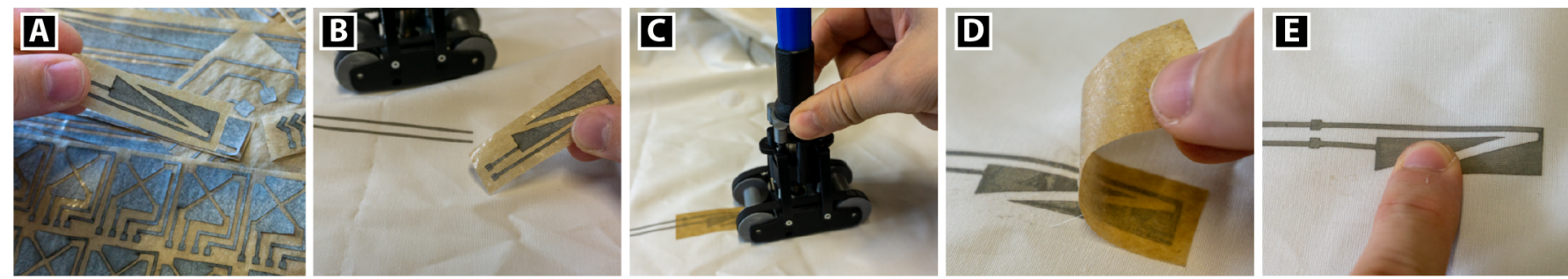

Figure 3. A selection of different patch modules showing various pre-manufactured functional multi-layer patches (A) and the application of a smallsized capacitive slider (B-E).

bles how an audiotape moves in-between two spools. Since tapes consist of iron-on electronics parts that are placed on a sticky, heat-resistant carrier layer, pushing down the heatingblock with the handle starts the iron-bonding (Figure 2, C). While moving the device on the textile, the continuous material is dispensed and ironed-on at the same time. The movement can be relatively quick, while very fast motions $>5 \mathrm{~cm} / \mathrm{sec}$ will negatively affect the adhesive and could potentially tear a tape. Ironing without any motion can damage the tape due to overheating. The maximum tape radius and length is currently defined by the wheels $(\mathrm{r}=12.5 \mathrm{~mm})$. However, tapes could also be placed on an independent axis above the wheels to support larger tapes and other form factors in future device iterations.

In order to steadily feed the device, we devised with a tape winding mechanism that is based on synchronized wheels using timing belts (see Figure 2, A). They also provide a pleasant rubber grip on the base textile surface which facilitates a precise hand-controlled sketching-like movement. Both timing belts move independently to facilitate angular motions. This allows for sketching curved shapes within the limits of the tape material that deforms during curved movement. In order to support automated tape cutting, we also integrated a micro DC gear motor with a linear actuator on which we mounted a small cutting blade (see Figure 2, D). It cuts the iron-on material to the desired length and does not cut the tape's carrier layer. A small push-button triggers cutting the tape at a desired location. All parts of the dispenser tool ${ }^{1}$ are 3D-printed with high temperature ABS filament [67] and partly protected with Teflon heat resistant fiberglass tape.

\section{Iron-On Material Stack}

In the following we will answer the questions, what RIO tapes and patches are actually made of and why they can be easily ironed on. In principle, each tape or patch consists of three layers: (1) an adhesive layer to bond it to the base textile, (2) a functional layer with a wide range of functional and also nonfunctional materials, and (3) a carrier Layer for holding the layout of the functional material in position until it is actually transferred to the textile.

\section{1 - Adhesive Layer}

To bond RIO tapes and patches to the base textile, we use double-sided iron-on adhesive consisting of thin fusible interlinings [68]. These adhesives are designed for fabric applications and thereby offer strong adhesion, washability and elastic deformation (cf. [68]). However, their adhesive power is less strong on smooth surfaces, like on flex PCBs. On such

\footnotetext{
${ }^{1}$ See https://www.imld.de/RIO/ for all technical details and parts.
}

surfaces, we add an ultra-thin adhesive layer with all-purpose glue [27] to the iron-on adhesive.

\section{2 - Functional Layer}

Iron-on tapes and patches can contain conductive materials. We use ultra-thin $(110 \mu \mathrm{m})$ tin, copper or silver plated nylon fabrics [59] for high-conductance elements (surface resistivity: $<0.02 \Omega / \mathrm{m}$ ). We furthermore use resistive [21] as well as piezoresistive [69] $(5-200 \mathrm{~N} ; 10-2,000 \Omega)$ fabrics. For isolating and dielectric elements, we use inkjet-printable textile transfer film [60]. In addition of using functional fabrics, we have successfully integrated printed electronics that are inkjetprinted [38] or screen-printed [46] using functional inks (e.g., conductive, dielectric, illuminating or thermochromic).

Finally, we demonstrate that commercial flexible electronic components can be integrated into iron-on tapes and patches. We demonstrate this for bendable microcontrollers [64], EL wires or organic solar cells [32].

We understand these raw materials (Figure 4) as dynamic building blocks and tapes that enable the creation of versatile custom I/O modules as we will present later. All of our used materials are representatives of their functional classes and can be easily extended or replaced with similar fabrics from other manufacturers (see [58] for an overview).

\section{3 - Carrier Layer}

To hold the functional part (e.g., multi-wire traces) of a patch in position until it is transferred to the textile, we use heat resistant baking paper that we coated with easily removable sticky glue [11] (see Figure 3, A for examples). This allows to safely roll tape designs on exchangeable material spools, to

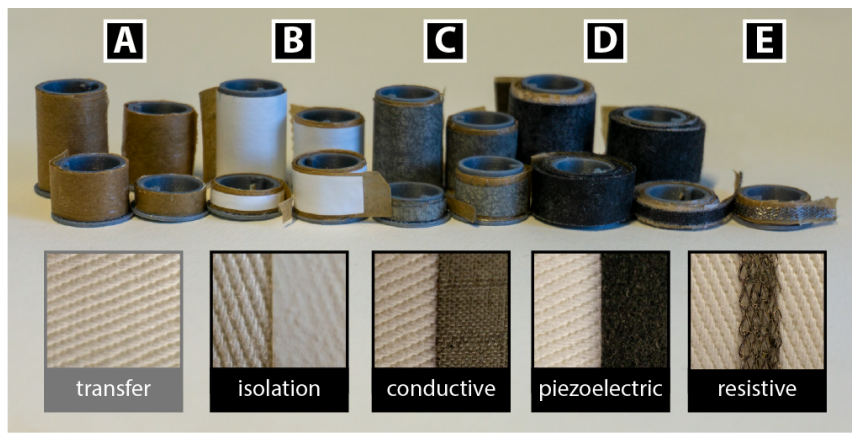

Figure 4. Different raw materials can be used for various applications. For instance, a generic transfer (A) can iron-on other materials, isolations (B) can shield or hide circuits, while conductive (C), piezoelectric (D) and resistive (E) fabric can be utilized for traces and sensors. 
transfer pre-manufactured tapes or patches without destroying their layout, and to easily peel it off after ironing (Figure 3, D).

In order to produce functional RIO modules, we first bond the previously introduced materials with heat-activatable bonding adhesives and subsequently cut all required tapes and patch parts using a vinyl or laser cutter.

\section{Composition Techniques}

By using continuous tapes and functional patches, the rapid iron-on approach enables a variety of composition techniques.

\section{Arranging}

First of all, designers have the freedom to arrange functional tapes freely in a sketching-like way. They can be combined with patches that are simply placed at the required position, ironed-on and then electrically connected in one stroke. While tapes can be arranged side by side manually, there are cases where a certain arrangement is repeatedly needed. For instance, a bus requires multiple parallel traces, or a continuous bend sensor might be aligned with a visual output tape.

Our RIO tool therefore supports custom tape assemblies that allow to seamlessly combine tapes side by side. Spools with tapes of different width and material can be stacked on a wheel and inserted into the tool (see Figure 1, C). This enables to iron on all required components side-by-side in one stroke.

\section{Layering}

Secondly, our approach supports versatile layering options to create new functionalities (e.g., shielded traces, multi-layer circuits or custom-shaped sensors by ironing multiple material layers on each other ) or to combine existing functions by ironing functional modules one above the other (e.g., combining visual output and touch tapes at the same position). Since our materials are ultra-thin and flexible, the capability of layering is one of the major advantages over existing approaches that use yarn or threads to isolate or extend conductors.

\section{Utilizing Textile Accessories}

Finally, our approach supports iron-on compositions that make use of existing textile accessories like zippers or buttons. We introduce the idea of utilizing textile landmarks and physical accessories by providing suitable iron-on tapes and patches to enhance textile functions with digital functions in a dual-use way. For this purpose, conductive textile accessories can be used as trace conductors by connecting them to a functional tape or patch. As an example, traces could be literally ironed to the teeth of zippers to physically bridge traces (see Figure 8). Another example is the four-way slider button patch using a cuff button discussed later (Figure 10, A3 and Figure 11, A).

\section{TRACES}

The flexibility to create conductive traces of custom length, direction and shape is a primary requirement. Moreover, traces are characterized by various additional properties for customization: level of conductivity, number of parallel wires (e.g., as required for bus systems), presence or absence of electrical shielding, stretchability and its connector pitches. In this section, we demonstrate how our iron-on device works in concert with iron-on trace spools to create basic conductive traces or traces with advanced multi-wire, elastic and shielded properties. We further present methods to create, connect and delete complex trace designs and realize custom-shaped sensor matrices using ironing and composition techniques.

\section{Creating Traces}

\section{Single-Wire and Multi-Wire Traces}

A spool for single wire traces is composed of a straight piece of iron-on conductive material, laser-cut to a width of $1.75 \mathrm{~mm}$, and affixed onto a carrier layer with sticky glue. The trace is applied using the rapid iron-on tool (see Figure 5) and can be ironed with a speed of approximately $2 \mathrm{~cm} /$ second. We measure an average conductivity of $0.1 \Omega / 1 \mathrm{~cm}$ for traces with a width of $1.75 \mathrm{~mm}$. This structure can be easily applied to almost all adhesive conductive fabrics, of different widths, or made of other materials (e.g., piezoresistive). We foresee that these spools and patches may be made commercially available, similarly to the wide variety of commodity textile crafting accessories that are already available today.

In addition, the same approach also supports rapidly creating traces that contain multiple parallel wires. For instance, this is required for connecting resistive sensors (e.g., pressure or bend) or multi-electrode capacitive sensors like a touch slider (see Figure 3). To do so, the designer has to select and load a multi-wire spool (see Figure 6) that contains multiple parallel conductors instead of just one. Our current implementation of the rapid iron-on device supports spools with a width of $23 \mathrm{~mm}$ supporting four wires aligned with a $5.08 \mathrm{~mm}$ pitch or eight wires based on a $2.54 \mathrm{~mm}$ pitch. Due to the textile substrate and the possible minimal offset during ironing, we decided to use the $5.08 \mathrm{~mm}$ pitch as a default for our approach.

\section{Elastic Traces}

An important, yet challenging, requirement for textile traces is their seamless integration into stretchable fabric. We support stretchability at the conductor-level by incorporating horseshoe pattern designs [23]. These are well-known to considerably enhance the stretchability of metallic conductors.

The RIO fabrication approach is compatible with several approaches: We started by ironing elastic conductor tapes from a smart textile manufacturer [66] that consists of stretchable yarns with integrated metallic conductors (Figure 6, B). While the stretchability and conductivity results are promising, the overall thickness $(8 \mathrm{~mm})$ of these elastic traces is quite large. Therefore, we contribute ultra-thin $(110 \mu \mathrm{m})$ elastic traces that are stretchable (up to $40 \%$ ) by using conductive iron-on material that we laser-cut with a horse-shoe pattern (Figure 1, D).
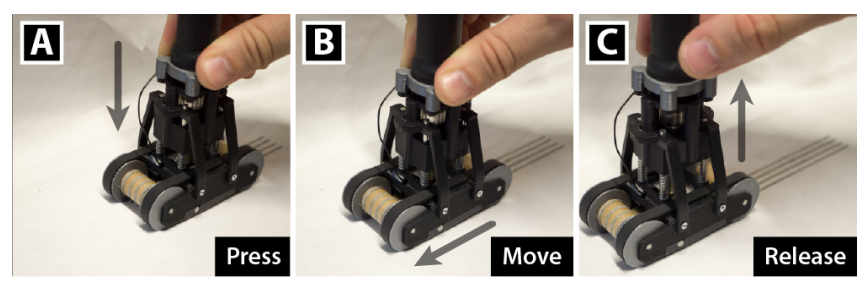

Figure 5. To create functional traces, the user has to press the materialloaded RIO device down (A), hold \& move the device in the desired direction $(B)$ and finally release the iron to finish $(C)$. 


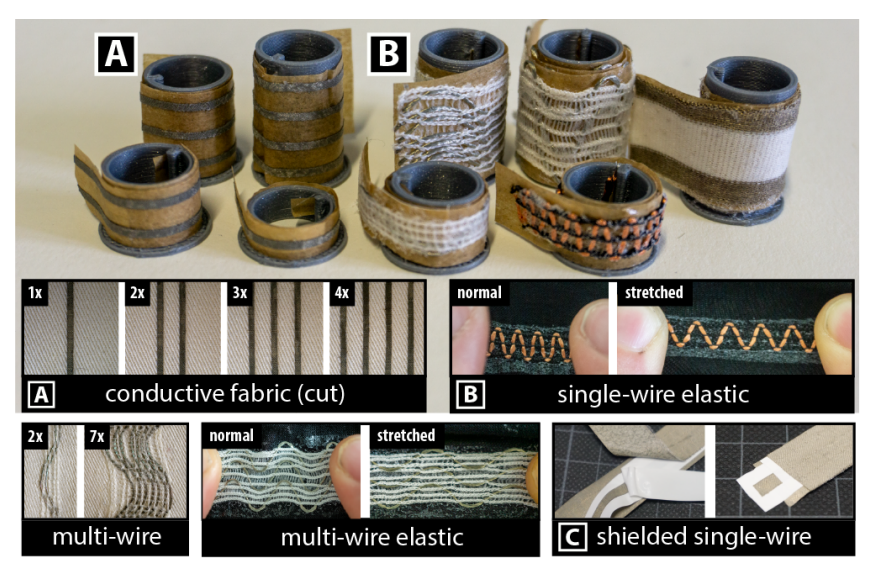

Figure 6. The rapid iron-on approach supports different types of traces, offering various design options regarding the number of wires, their thickness and stretchability.

\section{Isolated Traces}

In order to prevent electrical short circuits or unintended bridging caused by textile deformations, isolation tapes (see Figure $4, \mathrm{~B}$ ) physically insulate and protect traces. Therefore, we use a commercially available textile transfer films from skullpaper [60] as isolation material that combines adhesive properties with a polymer film in a thin $(10 \mu \mathrm{m})$ and flexible form factor. Therefore, isolated traces only add a total thickness of $120 \mu \mathrm{m}$ on the fabric substrate.

By choosing top-layer materials with appropriate thickness, stiffness or visio-haptic appearance, the same approach can be used to cover and hide traces or to enhance their robustness by mechanically strengthening them. The whole layer stack is dispensed at once, hence there is no need to add a separate isolation layer after having created the conductive trace.

\section{Shielded Traces}

Some textile circuits require electrical shielding of traces, e.g., for connecting to capacitive sensors or flexible antennas. Shielded traces are made of a stack of conductive $(110 \mu \mathrm{m})$ [59] and isolating $(10 \mu \mathrm{m})$ [60] fabrics preserving ultra-thin $(350 \mu \mathrm{m})$ and flexible properties (Figure 4, C).

\section{Connecting Traces}

More complex circuits can be readily created by connecting two or more traces.

\section{Extending Traces}

The easiest way to extend an existing trace is to start creating a new one on top that overlaps with an existing one. Since the heat-activatable tape adhesives consists of fusible structures that did not completely isolate the bonded surfaces when melt, bonded conductive materials will have a strong physical connection without a loss in conductivity. Therefore, the designer places the dispenser at the required position and moves the iron-on device in the desired direction. For individual wires, this method supports straight or angled connections. This approach also works for connecting multi-wire traces, as long as it follows the direction of the original multi-wire trace.

In a technical experiment we validated the end-to-end conductivity across a connection between traces. We created a continuous $40 \mathrm{~cm}$ long reference trace. For comparison, we created a second trace of the same overall length, which however consisted of 10 separate trace segments with overlapping ends, hence 9 electrical connections. Measurements showed that the resistance of both traces was equal $(4.1 \Omega)$.

\section{Create Angles and Crossings for Multi-Wire Traces}

To offer easy support for connecting two multi-wire traces at an angle, our toolkit makes use of dedicated connection patches. This special patch is made of electrically isolating material, but contains a slit for direct connection of individual conductive lines of multi-wire traces. The patch is shown in Figure 7, B. It is ironed onto an existing multi-wire trace at a location where it should connect to another one. After ironing a second multi-wire trace at an angle (Figure 7, E), each of the parallel wires electrically connects to its counterpart. As a result all traces are bridged and a highly conductive connection is established. Connections at a different angle, e.g., $45^{\circ}$, are realized by aligning the connection patch at a different angle.

\section{Reversible, On-demand Connection using Zipper}

By integrating iron-on circuits with a zipper, we demonstrate a reversible connection of multiple conductive lines. Figure 8 shows an example that connects eleven conductive lines of a detachable LilyPad to a textile circuit on a second textile patch, which can be attached or detached on-demand. Thereby, we show how the established principle of electrical slide fastener (cf. [2]) can be fabricated in an easy and elegant way without the need of large-scale zipper processing machinery.

One of the main rationales behind our implementation is the surprising finding that some metal zippers (e.g., [25]) only have a conductive connection to their opposite teeth, while not to adjacent ones, even when closed. To fabricate an ironon connection zipper, the designer first irons-on a conductor
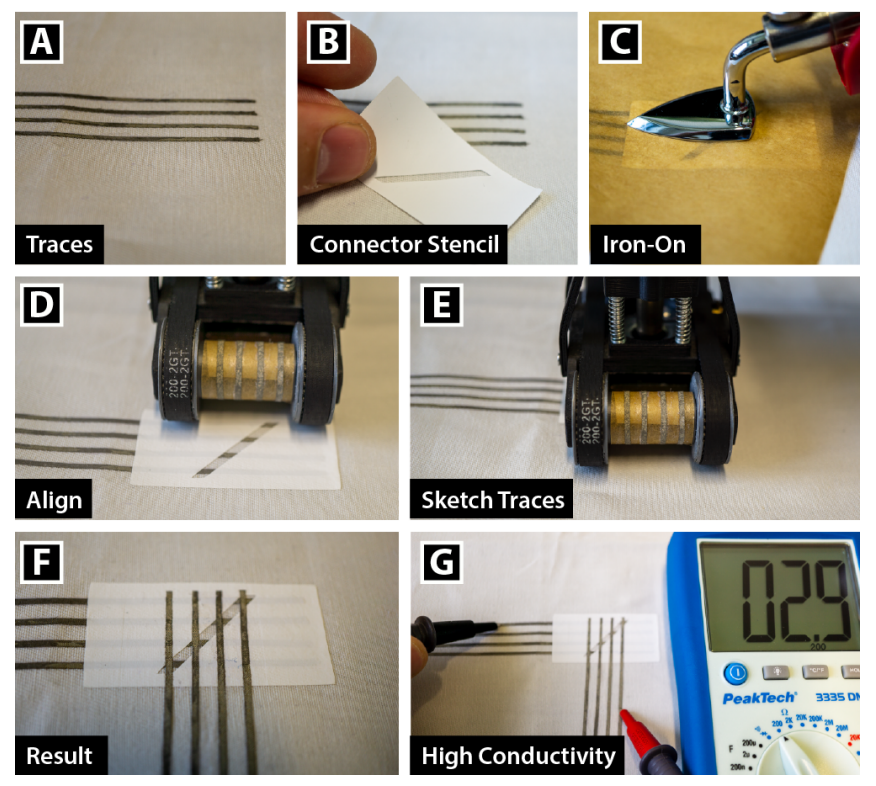

Figure 7. If a user wants to attach an angled connection to existing traces (A), a connector stencil (B) could be placed above and ironedon (C). Afterwards, our RIO tool could be moved above the stencil (D+E). The front axis previews the tape's alignment. As a result (F) all traces are bridged building a high conductive $(2.9 \Omega)$ connection $(\mathrm{G})$. 
patch aligned along the zipper. The patch splits the standard iron-on pitch $(5.08 \mathrm{~mm})$ to a larger pitch $(9.25 \mathrm{~mm})$ that aligns the conductors to every fourth zipper tooth. We thereby ensure non-shortcircuit connections. As a last step, the required zipper teeth have to be hooked-up manually by pulling the fabric iron-on conductors with a slit above the respective trace lines to ensure good conductivity. To avoid loose contacts, we added very small solder points at the teeth which strengthen the connection while not influencing a smooth fastening. This prototype demonstrates that iron-on circuits can be seamlessly integrated with commodity fashion accessories.

\section{Bridging Traces}

For bridging traces a thin isolation patch or tape (see Figure 4, B) can be placed at the required position to physically cover the conductive traces with a non-conductive iron-on film [60].

\section{Deleting Traces}

Mistakes happen or prototypes may evolve; hence changes may become necessary. All of our ironed components can be peeled off by ironing the parts again, to heat up the adhesive, and then using a hand or tweezers to carefully lift off the component. Alternatively to removing a conductive line, it can be selectively made non-conductive to remove current flow at a desired location. To do so, the iron is kept at this location for 5 seconds with a temperature of $250^{\circ} \mathrm{C}$. This leverages the property of the coating of the conductive fabric [59] to loose its conductivity when heated up to a high temperature.

\section{Sketching Custom-shaped Sensor Matrices with Traces}

To show the versatility of our techniques for creating and connecting traces, we demonstrate a custom-shaped pressure sensing matrix. Custom-shaped sensors are promising in textile electronics, as they can fit with the non-rectangular designs of clothing. The sensor is fabricated by applying three layers:

First, the bottom lines of the matrix are created in the desired size and (curved) shape (see Figure 9, A for an example with four parallel traces). Next, a flexible dielectric [60] or piezoelectric overlay [20] is added using the corresponding type of spool (B). Finally, the sensor is completed by sketching the crossing top lines of the matrix (C). The designer finishes the sensor by applying an isolation layer. Different types of multiplexed sensor matrices are possible, including multi-touch or pressure-touch matrices. We demonstrate a pressure-touch matrix using piezoresistive sensing. Therefore, a multiplexed data

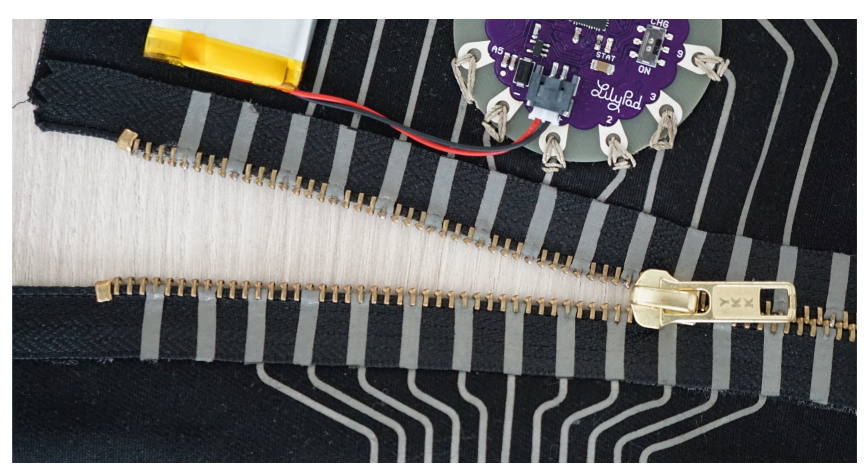

Figure 8. Zippers can be used to physically bridge fabrics with reversible connections.
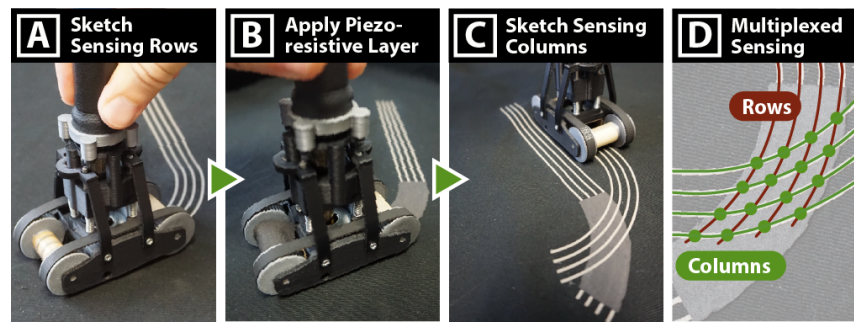

Figure 9. Pressure-Touch sensing matrix consisting of $4 \times 4$ electrodes and piezoresistive fabric.

acquisition chip (e.g., a 16-channel analog multiplexer [33]) measures the exercised force based on the resistive change of each electrode allowing to capture the distribution of pressure in the respective area.

\section{LIBRARY OF COMPONENTS}

We now demonstrate the feasibility of integrating various functional textile materials, printed electronics components and flexible PCBs inside iron-on tapes and patches. This allows us to leverage on a rich set of established principles for realizing functional iron-on components for both novices and advanced users. As selection criteria for identifying suitable iron-on components, we focus on the material parameters thickness, flexibility and temporal thermal resistance. We contribute a comprehensive library of components, both in tape and patch form factors, that offer advanced support for on-textile input, output, wiring, energy supply and computing. Figure 10 depicts an overview of all components.

\section{Input: Touch, Slide, Bend, Identify, Sense}

Capacitive touch input is realized by using a stack of conductive fabric [59] with iron-on adhesive. If required an additional shielding can be applied, resulting in a total patch thickness of 110 microns. Touch buttons (see Figure 10, A1) are discrete patches, can be ironed onto the desired location on the textiles and connected using shielded traces. Slider modules exist in a continuous tape form factor, based on interdigitated electrodes (cf. [61]), or as discrete patches of pre-defined size, using a triangular design with two electrodes [61].

Physical button controls leverage the tangible nature of commonplace textile accessories for input [15]. To demonstrate the benefits of integrating iron-on circuits with physical accessories, we realized one example: a four-way slider button patch. It is based on the classical four-electrode (cf. [56]) design $(25 \times 25 \mathrm{~mm})$ with an overlaid moveable conductor that is commonly used for measuring displacement. The upper, moveable layer is realized with fabric that is more stretchable than the lower layer. A standard metallic button for textiles can be affixed to it, acting as upper electrode and input control. When touched and moved by the user, the electrodes on the lower layer measure relative changes in capacitance, allowing to approximate the direction and extent of movement.

Piezoresistive pressure and bend input is implemented in tapes and patches that contain piezoresistive fabric [69] sandwiched in-between two conductive electrodes. The tape has two continuous lines for electrical connection on the left and on the right. Further commercially available components can be easily integrated into iron-on patches. We demonstrate this with 


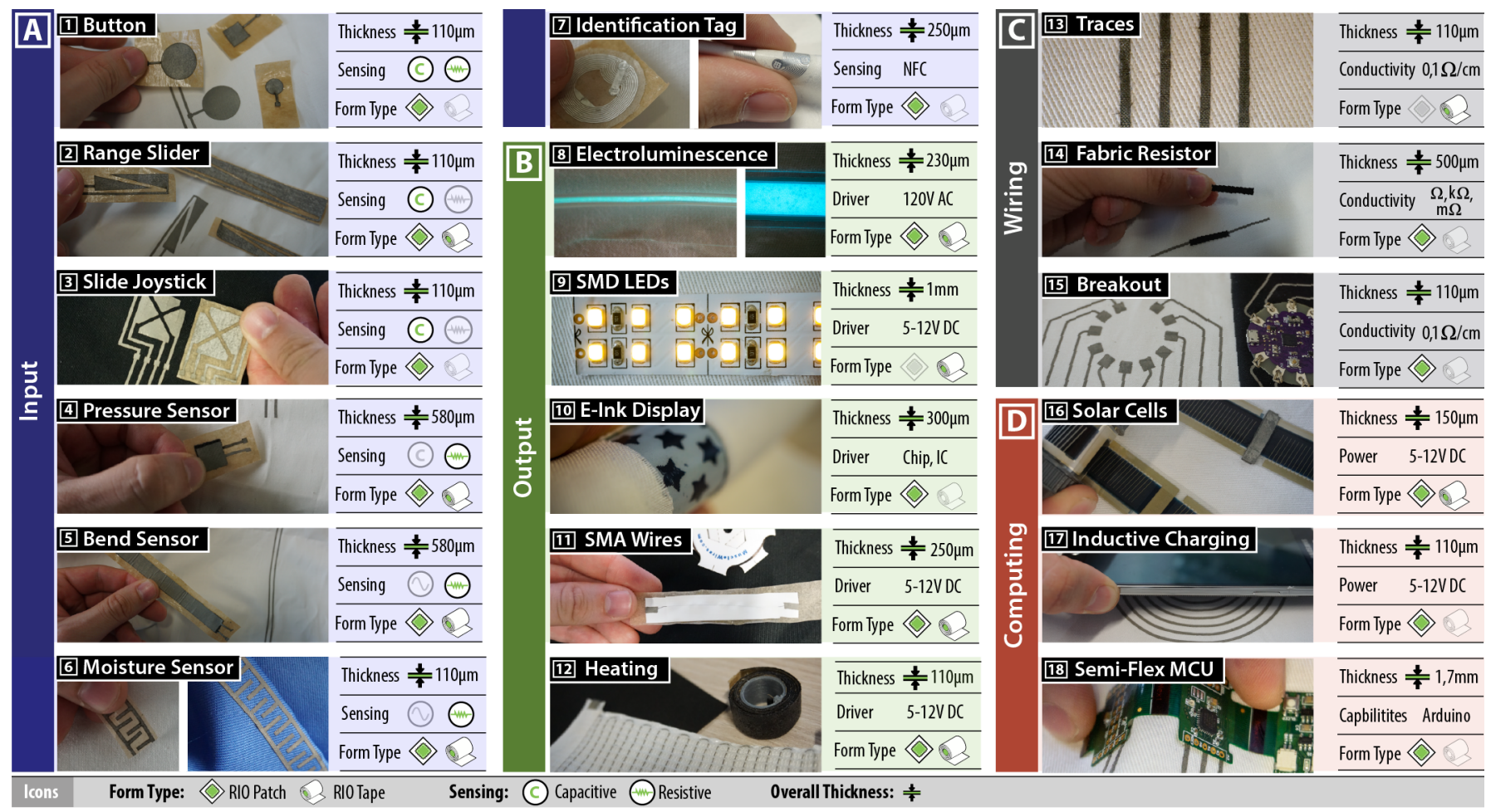

Figure 10. The comprehensive library of components consists of functional tapes and patches for input (A), output (B), wiring (C), and computing (D).

textile moisture [69] sensors. Measuring the wetness could be used, for instance, to detect the context like a rainy day or specific events such as the recognition when a drinking bottle is leaking in a backpack. Similarly, we augment ultra-thin NFC tags [44] with iron-on adhesive [68], allowing the user to simply place the tag and iron it onto the textile.

\section{Output: Visual, Haptic and Shape-Change}

In order to explore the possibilities of visual feedback, we demonstrate the integration of three types of visual output with different degrees of integration and complexity.

Electro-luminescent wires, tapes and patches offer segmentbased or line-shaped illumination and are very deformable, offering outstanding textile compatibility. Off-the-shelf EL wires with welted piping [1] are integrated into the RIO tool by using an L-shaped iron (see Figure 2, E4). Alternatively, a flexible EL display could be screen-printed [46] onto a flexible adhesive tape or patch to create free-form segment displays.

As a second option, conventional SMD-LED tapes can be used. They offer rich capabilities such as individual addressable, bright and full-color LEDs, however, they are less deformable. By using our U-shaped iron (see Figure 2, E2) it is further possible to iron-on semi-flex LED stripes with partly rigid components. Therefore, the iron only heats up the lateral edges of the tape and melts the adhesive to the fabric, preventing the delicate electronic components to overheat. This is an example how the RIO approach also allows to integrate semi-flex SMD.

Finally, prior research has shown that $e$-ink displays have great potential for use with textiles (cf. [17]). Therefore, we use bendable e-ink displays [18] to fabricate patches (Figure 10, B10) that can be ironed and connected with a multi-wire tape.
Besides visual output, the rapid iron-on approach also supports seamless integration of haptic or shape-changing output inside a textile prototype.

Thermal output is realized by integrating resistive heating fabric that heats up $\left(45-60^{\circ} \mathrm{C}\right)$ when a low-voltage $(\sim 5 \mathrm{~V}[12]$, $12 \mathrm{~V}[21])$ current is applied. It preserves thin, soft and deformable properties. Since the maximum heating temperature of the patches is $60^{\circ} \mathrm{C}$ the patch adhesive does not melt.

To realize shape-change, we integrated shape memory alloys (SMA). We combine Dynalloy Flexinol [19] with a carrier fabric to provide a thin $(d=150 \mu \mathrm{m})$ and noticeable $(f=321 g)$ actuation with low current $(A=410 \mathrm{~mA})$.

For integrating vibro-tactile output in a slim, deformable form factor, we use noiseless micro-actuators made from a $0.13 \mathrm{~mm}$ thick flexible PCB. We build on the design from Bugeja [8] and extend it with heat-activated adhesive and a magnetic counterpart. This realizes a coreless flexible electromagnet, that can attract or repel a neodymium magnet. With this combination the designer is able to create either very subtle punctually sensations or flutter effects of fabric pieces.

\section{Computing: Powering and Processing}

We propose to power up a circuit by harvesting solar energy from the environment. Therefore, we use printed solar cell tapes [32] that are flexible and can be cut at any location. We added heat-activatable adhesive to make the tape suitable for our rapid iron-on dispenser device. Depending on the design, sketched lines or even rectangle assemblies consisting of multiple lines are possible. Note that it is important to consider the direction of each solar tape for a positive voltage potential. The solar cell is connected to the circuit through a charging 

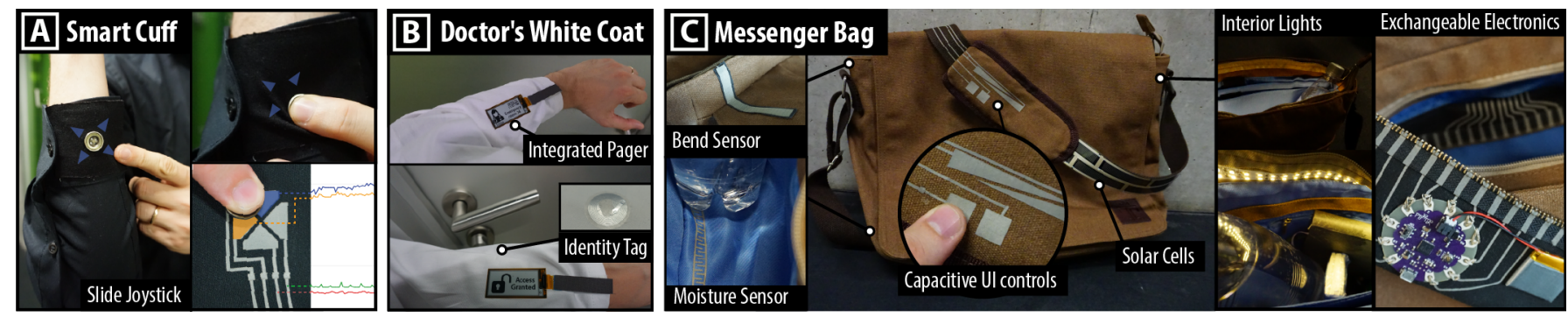

Figure 11. We present the three application examples of a Smart Cuff for Business Presentations (A), a Doctor's White Coat (B) and a Messenger Bag (C).

board with a LiPo battery [32]. While best performance (8$9 \mathrm{~V} / \mathrm{m} ; 40-50 \mathrm{~mA})$ is achieved when the solar cell is added to the textile's outer side, it is worth noting that many nondensely woven fabrics (e.g., cotton) let pass light. This allows for adding the solar cell on the inner side, making it invisible.

Wireless power transfer through inductive charging is enabled through iron-on patches with an integrated charging coil made of conductive fabric [65]. The designer can, for instance, iron-on a charging coil patch inside a pocket; whenever the user puts a smartphone with inductive charging capabilities into the pocket, the textile circuit is powered. Further, ironedon charging coils that are made of conductive fabric enable the wireless power transfer through an inductive coupling at a short range. Bidirectional wireless inductive powering in mobiles (e.g., Samsung Wireless PowerShare [57]) could be used to power smart garments.

Processing: Our platform supports many microcontrollers. Arduino LilyPad is frequently used for textile prototypes. For integrating a LilyPad without sewing, we contribute a patch that can be zipped onto a textile circuit. It uses the zipper connection demonstrated. The patch contains one side of a zipper that connects to the pins of the LilyPad (see Figure 8).

\section{EXAMPLE APPLICATIONS}

To demonstrate the versatility of the rapid iron-on fabrication approach, we used it to implement three example applications for ubiquitous and wearable computing.

A Smart Cuff (see Figure 11, A) acts as a direct and discreet input device, e.g., for controlling a slide deck during a business presentation. It demonstrates how existing garments can be easily augmented using functional iron-on patches. We used a four-way button patch from our library of components (see Figure 10, A3). It realizes an interactive metal button that can be slightly displaced in all directions and snaps back elastically, while sensing its position. A four-wire shielded trace is used for connection.

The second application example is a Doctor's White Coat (see Figure 11, B), demonstrating how advanced electronics can be easily integrated by ironing. The coat helps medical staff to quickly get access to secured areas by using a sleeve-integrated RFID patch for quick identification. In addition, it contains an e-ink patch that can visualize important pager notifications.

An interactive Messenger Bag (see Figure 11, C) demonstrates how complex textile products with non-planar surfaces can be enhanced with smart functions. We integrated various smart functions including interior lights that illuminate when the bag is open, moisture sensors at the bag's bottom that notify its wearer when a bottle leaks, capacitive buttons and slider controls at the shoulder pad for controlling a music player, and solar cells for power harvesting. We started by ironing the solar cells and trace electronics at the belt of the bag. Due to its linear form factor our rapid iron-on device worked easily on this substrate. However, some parts of the bag, like its inside, were more challenging to realize since they did not provide areas we could easily iron on. Therefore, we turned the blue inner lining to the outside and were able to apply the entire moisture sensor with one stroke. Furthermore, the bag lights up when it is open. For that, we used SMD-LEDs and a bend sensor that we ironed in the bag.

\section{EXPERT REVIEWS}

To gain insights regarding the suitability of the RIO approach within the domain of textiles, garments and fashion, we conducted hands-on reviews and interviews with professional experts in fashion and textile design as well as textile machinery.

\section{Textile \& Fashion Design Experts}

Our first expert (P1, co-director of the textile \& materials design department of an internationally recognized French design university) has both a design and research background and does interdisciplinary work on textile design, architecture and new technologies. Our second expert (P2, PhD fashion design) has expertise in practice-based and design-led research investigating tailoring. We conducted two semi-structured interviews that each lasted around an hour. They comprised a hands-on session with representative samples, material spools and patches as well as the rapid iron-on device.

First of all, the textile expert (P1) and fashion expert (P2) highlighted the importance of smart garments and its design as a hot topic. P1 stated that »Companies will open up new markets for FashionTech « and emphasized that start-up, research and commercial innovation labs are already working on it. She stated that future everyday markets will appear and include smart sports and activity tracking fashion, textile medical products as well as interactive furniture and accessories. However, P1 also highlighted that textile and shape designers traditionally did not know anything about circuits, programming and technical challenges: »Electricity is not part of the framework of our thinking - it is new for us that fabrics became active materials «. Subsequently, we asked how FashionTech prototypes are currently being realized. Both experts reported that for interactive garment projects a technician must be in the on-site team, however, this often slows down the design process since a common vocabulary and collectively agreed-upon design 
have to be brought together. P2 stated that: »Textile \& shape designers and electrical engineers should better work together - otherwise stupid products will emerge".

In this line, both experts emphasized that the iron-on approach has a promising potential to empower designers to build their own design-driven prototypes at an early design stage, allowing them to better communicate their technical requirements to engineers. P2 commented: »The iron-on roll \& patch approach is in-between large-scale production and manual crafting - this makes absolutely sense «. Further, P2 appreciated the thin and flexible properties and rated the iron-on working principle as simple, convincing and a familiar manufacturing process. In contrast, she characterized sewing to be typically slower and requires more knowledge.

However, both experts (P1, P2) underlined the importance to work from the beginning with final materials to consider the visual and sensory qualities in the whole design process. While it was our initial intention that iron-on tapes and patches support the prototyping process, whereas a final exhibition prototype would be realized using different technology, P1 proposed to bridge this gap. Therefore, P1 recommended to include highly integrated and versatile (e.g., different colors) samples in the kit to provide a preview of the possible degree of integration and design options.

Further, the experts reported that our kit could be a great teaching tool for prototyping interactive textile and garments in classrooms and workshops. P1 commented that it is a "fantastic toolkit for students of fashion design and textile design«, requesting an online or offline documentation.

Finally, P2 proposed that non-expert users, such as members of the maker, crafting and DIY communities, could also use our iron-on kit to make their own smart clothes. Further, she suggested using low-temperature heat-activated adhesive to create temporal sticky designs for special events (e.g., business presentations or party events) that can be later removed by washing or repeated ironing.

\section{Textile Machinery \& Fiber Expert}

To further discuss our iron-on approach from a more technical perspective, we interviewed a fiber expert ( $\mathrm{P} 3$, research associate), working at the textile machinery research institute of Technische Universität Dresden. He has prior education in fashion design and a strong background in textile machinery, textile structures and fiber technologies. The session lasted one hour. It comprised a semi-structure interview as well as an interactive hands-on session in which P3 was asked to sketch conductive traces and apply selected patches by using our rapid iron-on device.

P3 stated that he personally knows dressmakers who make interactive costumes for theatre performances that are either controlled by hidden trigger cables or triggered by an assistant remotely. He highlighted the promising potential of the rapid iron-on kit in this context. While ironing-on traces onto regular fabric worked well, he raised concerns that the iron-on approach might not be suitable for precious and fine textiles like silk or cashmere. P3 commented that when used on stretchable fabric, the iron-on tool tended to slightly stretch the fabric while delivering traces. Caused by the wheels' friction, this created a slight material deformation, which is not desirable for high-end textiles.

Finally, we discussed several metrics by using a radar chart with six axes to compare the RIO approach with other e-textile solutions like embroidery and knitting. As a result, P3 highlighted that the RIO approach has clear advantages along the axes of production time, required knowledge and costs, is comparable regarding the scope of supported functionality and has disadvantages in its durability and degree of integration.

\section{Discussion and Limitations}

Overall, we received positive feedback from the experts for our concept and its current implementation, however, the interviews also revealed limitations. The interviews confirmed the need for rapid prototyping tools for smart fashion and textile designers, offering support in an early design stage. We learned that the designer value the flexible and thin components of our iron-on kit and its versatile application possibilities. However, we also gained insights that textile and fashion designer would still prefer classical prototypes for final show garments due to the compatibility with a wider set of textile materials. Future work should investigate how these can be integrated with iron-on tapes and patches.

Further limitations of our current realization consist of the limited curve radius that can be achieved with our current tool when creating continuous traces. Future iterations can solve this issue by offering a smaller handheld iron-on dispenser.

\section{CONCLUSION \& FUTURE WORK}

We presented Rapid Iron-on User Interfaces, a novel fabrication approach for interactive textile prototypes that allows to enhance fabrics with rich digital functionalities. By introducing rolled iron-on tapes and a rich set of patches, made of functional materials, thin-film printed electronics and flexible components, we discussed how a handheld dispenser tool enables rapid fabrication in a sketching-like fashion. With its accompanying comprehensive library of heat-activated adhesive functional tapes and patches, we demonstrated the technical feasibility of this approach and its widespread applicability. We further demonstrated the versatility of our toolkit by implementing applications for ubiquitous and wearable computing. Finally, findings from an expert review identified benefits, limitations and opportunities for future work. For future work, we plan to run multi-day workshops in design schools and maker spaces to investigate adoption and usage practice with textile students and makers.

\section{Acknowledgments}

This work was funded in part by DFG grant 389792660 as part of TRR 248, and the DFG as part of Germany's Excellence Strategy EXC 2050/1 - Project ID 390696704 - Cluster of Excellence "Centre for Tactile Internet with Human-in-theLoop" (CeTI) of Technische Universität Dresden. In addition, this project received funding from the European Research Council (ERC StG Interactive Skin 714797). 


\section{REFERENCES}

[1] Adafruit. 2019. Sewable Electroluminscent (EL) Wire Welted Piping- Aqua 5 meters. (2019).

https://www. adafruit . com/product/675

[2] Robert W Avery. 1953. Electrical slide fastener connector. (Mar 1953).

https://patents. google. com/patent/US2877439A/

[3] Senem Kurşun Bahadir and Umut Kivanc Sahin. 2018. A Wearable Heating System with a Controllable e-Textile- Based Thermal Panel. In Wearable Technologies, Jesús Hamilton Ortiz (Ed.). IntechOpen, Rijeka, Chapter 9, 175-194. DOI :

http://dx.doi.org/10.5772/intechopen.76192

[4] E. Bonderover and S. Wagner. 2004. A woven inverter circuit for e-textile applications. IEEE Electron Device Letters 25, 5 (May 2004), 295-297. DOI : http://dx.doi.org/10.1109/LED.2004.826537

[5] L. Buechley and M. Eisenberg. 2008. The LilyPad Arduino: Toward Wearable Engineering for Everyone. IEEE Pervasive Computing 7, 2 (April 2008), 12-15. DOI : http://dx. doi .org/10.1109/MPRV.2008.38

[6] Leah Buechley and Michael Eisenberg. 2009. Fabric PCBs, Electronic Sequins, and Socket Buttons: Techniques for e-Textile Craft. Personal Ubiquitous Comput. 13, 2 (Feb. 2009), 133-150. DOI : http://dx.doi.org/10.1007/s00779-007-0181-0

[7] Leah Buechley, Mike Eisenberg, Jaime Catchen, and Ali Crockett. 2008. The LilyPad Arduino: Using Computational Textiles to Investigate Engagement, Aesthetics, and Diversity in Computer Science Education. In Proceedings of the SIGCHI Conference on Human Factors in Computing Systems (CHI '08). ACM, New York, NY, USA, 423-432. DOI:

http://dx.doi.org/10.1145/1357054.1357123

[8] Carl Bugeja. 2019. Flexible PCB Actuator - The thinnest Magnetic Actuator! (2019). https://www.tindie.com/ products/Microbots/flexible-pcb-actuator/

[9] Justin Chan and Shyamnath Gollakota. 2017. Data Storage and Interaction Using Magnetized Fabric. In Proceedings of the 30th Annual ACM Symposium on User Interface Software and Technology (UIST '17). ACM, New York, NY, USA, 655-663. DOI : http://dx.doi.org/10.1145/3126594.3126620

[10] Jun Chen, Yi Huang, Nannan Zhang, Haiyang Zou, Ruiyuan Liu, Changyuan Tao, Xing Fan, and Zhong Lin Wang. 2016. Micro-cable structured textile for simultaneously harvesting solar and mechanical energy. Nature Energy 1 (2016), 16138.

https://doi.org/10.1038/nenergy.2016.138 Article.

[11] Kuretake Co., Ltd. 2019a. Zig Memory System - 2-Way Glue Pen. MSB-30M. (2019).

https://www.kuretake.co.jp/en/

[12] WireKinetics Co., Ltd. 2019b. Ultra Heating Fabric. (2019). https:

//www. wirekinetics.com/en/proList.php?class_sn=3
[13] Joshua DeGraff, Richard Liang, Minh Quyen Le, Jean-Fabien Capsal, Florent Ganet, and Pierre-Jean Cottinet. 2017. Printable low-cost and flexible carbon nanotube buckypaper motion sensors. Materials \& Design 133 (2017), 47 - 53. DOI :

http://dx.doi.org/10.1016/j .matdes.2017.07.048

[14] Artem Dementyev, Hsin-Liu (Cindy) Kao, and Joseph A. Paradiso. 2015. SensorTape: Modular and Programmable 3D-Aware Dense Sensor Network on a Tape. In Proceedings of the 28th Annual ACM Symposium on User Interface Software \&\#38; Technology (UIST '15). ACM, New York, NY, USA, 649-658. DOI : http://dx.doi.org/10.1145/2807442.2807507

[15] Artem Dementyev, Tomás Vega Gálvez, and Alex Olwal. 2019. SensorSnaps: Integrating Wireless Sensor Nodes into Fabric Snap Fasteners for Textile Interfaces. In Proceedings of the 32Nd Annual ACM Symposium on User Interface Software and Technology (UIST '19). ACM, New York, NY, USA, 17-28. DOI : http://dx.doi.org/10.1145/3332165.3347913

[16] Laura Devendorf, Joanne Lo, Noura Howell, Jung Lin Lee, Nan-Wei Gong, M. Emre Karagozler, Shiho Fukuhara, Ivan Poupyrev, Eric Paulos, and Kimiko Ryokai. 2016. "I don't want to wear a screen": Probing Perceptions of and Possibilities for Dynamic Displays on Clothing. In Proceedings of the $2016 \mathrm{CHI}$ Conference on Human Factors in Computing Systems (CHI '16). ACM, New York, NY, USA, 6028-6039. DOI : http://dx . doi .org/10.1145/2858036.2858192

[17] Christine Dierk, Molly Jane Pearce Nicholas, and Eric Paulos. 2018. AlterWear: Battery-Free Wearable Displays for Opportunistic Interactions. In Proceedings of the 2018 CHI Conference on Human Factors in Computing Systems (CHI '18). ACM, New York, NY, USA, Article 220, 11 pages. DOI : http://dx.doi .org/10.1145/3173574.3173794

[18] Good Display. 2019. 2.13 Inch E-Paper Display, Flexible Partial Refresh, GDEW0213I5F. (2019). https://www. good-display.com/

[19] Inc DYNALLOY. 2019. FLEXINOL ${ }^{\circledR}$ Actuator Wire. (2019). http://www . dynalloy. com/flexinol.php

[20] EeonTex. 2019. EeonTex Conductive Stretchable Fabric. (2019).

https://www. sparkfun. com/products/retired/14112

[21] Eeonyx. 2019. Thermionyx Nonwoven Warming Fabric Polyester \& Nylon blend. (2019).

https://eeonyx. com/products/thermionyx-nonwoven/

[22] Yu Enokibori, Akihisa Suzuki, Hirotaka Mizuno, Yuuki Shimakami, and Kenji Mase. 2013. E-textile Pressure Sensor Based on Conductive Fiber and Its Structure. In Proceedings of the 2013 ACM Conference on Pervasive and Ubiquitous Computing Adjunct Publication (UbiComp '13 Adjunct). ACM, New York, NY, USA, 207-210. DOI :

http://dx.doi.org/10.1145/2494091.2494158 
[23] Jonathan A. Fan, Woon-Hong Yeo, Yewang Su, Yoshiaki Hattori, Woosik Lee, Sung-Young Jung, Yihui Zhang, Zhuangjian Liu, Huanyu Cheng, Leo Falgout, Mike Bajema, Todd Coleman, Dan Gregoire, Ryan J. Larsen, Yonggang Huang, and John A. Rogers. 2014. Fractal design concepts for stretchable electronics. Nature Communications 5, 1 (2014), 3266. DOI : http://dx.doi.org/10.1038/ncomms4266

[24] Jonny Farringdon, Andrew J. Moore, Nancy Tilbury, James Church, and Pieter D. Biemond. 1999. Wearable Sensor Badge and Sensor Jacket for Context Awareness. In Proceedings of the 3rd IEEE International Symposium on Wearable Computers (ISWC '99). IEEE Computer Society, Washington, DC, USA, 107-. http://dl. acm. org/citation. cfm?id=519309. 856485

[25] YKK Fastening. 2019. Metal Zipper, Standard 5RG, Golden Brass. (2019). https://www. ykkfastening. com/

[26] Mikhaila Friske, Shanel Wu, and Laura Devendorf. 2019. AdaCAD: Crafting Software For Smart Textiles Design. In Proceedings of the 2019 CHI Conference on Human Factors in Computing Systems (CHI '19). ACM, New York, NY, USA, Article 345, 13 pages. DOI: http://dx.doi.org/10.1145/3290605.3300575

[27] UHU All Purpose Adhesive Glue. 2019. Manufacturer Part Number: 40063. (2019). https://www. uhu . com

[28] Daniel Groeger and Jürgen Steimle. 2019. LASEC: Instant Fabrication of Stretchable Circuits Using a Laser Cutter. In Proceedings of the 2019 CHI Conference on Human Factors in Computing Systems (CHI'19). Association for Computing Machinery, New York, NY, USA, Article Paper 699, 14 pages. DOI :

http://dx.doi.org/10.1145/3290605.3300929

[29] Tovi Grossman, Ravin Balakrishnan, and Karan Singh. 2003. An Interface for Creating and Manipulating Curves Using a High Degree-of-freedom Curve Input Device. In Proceedings of the SIGCHI Conference on Human Factors in Computing Systems (CHI '03). ACM, New York, NY, USA, 185-192. DOI :

http://dx.doi.org/10.1145/642611.642645

[30] Nur Al-huda Hamdan, Simon Voelker, and Jan Borchers. 2018. Sketch\&Stitch: Interactive Embroidery for E-textiles. In Proceedings of the 2018 CHI Conference on Human Factors in Computing Systems (CHI '18). ACM, New York, NY, USA, Article 82, 13 pages. DOI : http://dx.doi .org/10.1145/3173574.3173656

[31] Megan Hofmann, Lea Albaugh, Ticha Sethapakadi, Jessica Hodgins, Scott E. Hudson, James McCann, and Jennifer Mankoff. 2019. KnitPicking Textures: Programming and Modifying Complex Knitted Textures for Machine and Hand Knitting. In Proceedings of the 32Nd Annual ACM Symposium on User Interface Software and Technology (UIST '19). ACM, New York, NY, USA, 5-16. DOI :

http://dx.doi.org/10.1145/3332165.3347886
[32] infinityPV. 2019. infinityPV Solar Tape. (2019). infinitypv.com

[33] Texas Instruments. 2019. High-speed CMOS 16-channel Analog Multiplexer/Demultiplexer. (2019). http://www.ti. com/product/CD74HC4067

[34] Alexandre Kaspar, Liane Makatura, and Wojciech Matusik. 2019. Knitting Skeletons: A Computer-Aided Design Tool for Shaping and Patterning of Knitted Garments. In Proceedings of the 32Nd Annual ACM Symposium on User Interface Software and Technology (UIST '19). ACM, New York, NY, USA, 53-65. DOI : http://dx.doi.org/10.1145/3332165.3347879

[35] Eva-Sophie Katterfeldt, Nadine Dittert, and Heidi Schelhowe. 2009. EduWear: Smart Textiles As Ways of Relating Computing Technology to Everyday Life. In Proceedings of the 8th International Conference on Interaction Design and Children (IDC '09). ACM, New York, NY, USA, 9-17. DOI :

http://dx.doi.org/10.1145/1551788.1551791

[36] Joshua Kaufman, Felix Tan, Morgan Monroe, and Ayman Abouraddy. 2018. User-Controlled Color-Changing Textiles: From Prototype to Mass Production. ICE 2018: International Conference on E-textiles, Paris, France, (Aug 27-28, 2018) 12, 8 (2018), 141.

[37] Majeed Kazemitabaar, Jason McPeak, Alexander Jiao, Liang He, Thomas Outing, and Jon E. Froehlich. 2017. MakerWear: A Tangible Approach to Interactive Wearable Creation for Children. In Proceedings of the 2017 CHI Conference on Human Factors in Computing Systems (CHI '17). ACM, New York, NY, USA, 133-145. DOI :

http://dx.doi.org/10.1145/3025453.3025887

[38] Arshad Khan, Joan Sol Roo, Tobias Kraus, and Jürgen Steimle. 2019. Soft Inkjet Circuits: Rapid Multi Material Fabrication of Soft Circuits Using a Commodity Inkjet Printer. In Proceedings of the 32th Annual ACM Symposium on User Interface Software and Technology (UIST '19). DOI:

http://dx.doi.org/10.1145/3332165.3347892

[39] Seonil Kwon, Hyuncheol Kim, Seungyeop Choi, Eun Gyo Jeong, Dohong Kim, Somin Lee, Ho Seung Lee, Young Cheol Seo, and Kyung Cheol Choi. 2018. Weavable and Highly Efficient Organic Light-Emitting Fibers for Wearable Electronics: A Scalable, Low-Temperature Process. Nano Letters 18, 1 (10 Jan 2018), 347-356. DOI :

http://dx.doi.org/10.1021/acs. nanolett.7b04204

[40] Jingyi Li, Jennifer Jacobs, Michelle Chang, and Björn Hartmann. 2017. Direct and Immediate Drawing with CNC Machines. In Proceedings of the 1st Annual ACM Symposium on Computational Fabrication (SCF' 17). ACM, New York, NY, USA, Article 11, 2 pages. DOI : http://dx.doi.org/10.1145/3083157.3096344 
[41] Janis Lena Meissner, Angelika Strohmayer, Peter Wright, and Geraldine Fitzpatrick. 2018. A

Schnittmuster for Crafting Context-Sensitive Toolkits. In Proceedings of the 2018 CHI Conference on Human Factors in Computing Systems (CHI'18). ACM, New York, NY, USA, Article 151, 151:1-151:13 pages. DOI : http://dx.doi.org/10.1145/3173574. 3173725

[42] Stefanie Mueller, Pedro Lopes, and Patrick Baudisch. 2012. Interactive Construction: Interactive Fabrication of Functional Mechanical Devices. In Proceedings of the 25th Annual ACM Symposium on User Interface Software and Technology (UIST'12). ACM, New York, NY, USA, 599-606. DOI :

http://dx.doi.org/10.1145/2380116.2380191

[43] Grace Ngai, Stephen C.F. Chan, Hong Va Leong, and Vincent T.Y. Ng. 2013. Designing I*CATch: A Multipurpose, Education-friendly Construction Kit for Physical and Wearable Computing. Trans. Comput. Educ. 13, 2, Article 7 (July 2013), 30 pages. DOI : http://dx.doi.org/10.1145/2483710.2483712

[44] NXP. 2019. MIFARE Classic 1K RFID NFC 25mm. (2019). https://www.nxp.com

[45] Simon Olberding, Nan-Wei Gong, John Tiab, Joseph A. Paradiso, and Jürgen Steimle. 2013. A Cuttable Multi-Touch Sensor. In Proceedings of the 26th Annual ACM Symposium on User Interface Software and Technology (UIST'13). Association for Computing Machinery, New York, NY, USA, 245-254. DOI : http://dx.doi.org/10.1145/2501988.2502048

[46] Simon Olberding, Michael Wessely, and Jürgen Steimle. 2014. PrintScreen: Fabricating Highly Customizable Thin-film Touch-displays. In Proceedings of the 27th Annual ACM Symposium on User Interface Software and Technology (UIST '14). ACM, New York, NY, USA, 281-290. DOI :

http://dx.doi.org/10.1145/2642918.2647413

[47] Maggie Orth, Rehmi Post, and Emily Cooper. 1998. Fabric Computing Interfaces. In CHI 98 Conference Summary on Human Factors in Computing Systems (CHI '98). ACM, New York, NY, USA, 331-332. DOI : http://dx.doi.org/10.1145/286498.286800

[48] Patrick Parzer, Florian Perteneder, Kathrin Probst, Christian Rendl, Joanne Leong, Sarah Schuetz, Anita Vogl, Reinhard Schwoediauer, Martin Kaltenbrunner, Siegfried Bauer, and Michael Haller. 2018. RESi: A Highly Flexible, Pressure-Sensitive, Imperceptible Textile Interface Based on Resistive Yarns. In Proceedings of the 31st Annual ACM Symposium on User Interface Software and Technology (UIST '18). ACM, New York, NY, USA, 745-756. DOI : http://dx.doi.org/10.1145/3242587.3242664

[49] Patrick Parzer, Adwait Sharma, Anita Vogl, Jürgen Steimle, Alex Olwal, and Michael Haller. 2017. SmartSleeve: Real-time Sensing of Surface and Deformation Gestures on Flexible, Interactive Textiles, Using a Hybrid Gesture Detection Pipeline. In
Proceedings of the 30th Annual ACM Symposium on User Interface Software and Technology (UIST'17). ACM, New York, NY, USA, 565-577. DOI : http://dx.doi.org/10.1145/3126594.3126652

[50] Andreas Peetz, Konstantin Klamka, and Raimund Dachselt. 2019. BodyHub: A Reconfigurable Wearable System for Clothing. In The Adjunct Publication of the 32Nd Annual ACM Symposium on User Interface Software and Technology (UIST'19). ACM, New York, NY, USA, 39-41. DOI :

http://dx.doi.org/10.1145/3332167.3357108

[51] Huaishu Peng, Jimmy Briggs, Cheng-Yao Wang, Kevin Guo, Joseph Kider, Stefanie Mueller, Patrick Baudisch, and François Guimbretière. 2018. RoMA: Interactive Fabrication with Augmented Reality and a Robotic 3D Printer. In Proceedings of the 2018 CHI Conference on Human Factors in Computing Systems (CHI'18). ACM, New York, NY, USA, Article 579, 12 pages. DOI : http://dx.doi.org/10.1145/3173574.3174153

[52] Huaishu Peng, Jennifer Mankoff, Scott E. Hudson, and James McCann. 2015. A Layered Fabric 3D Printer for Soft Interactive Objects. In Proceedings of the 33rd Annual ACM Conference on Human Factors in Computing Systems (CHI '15). ACM, New York, NY, USA, 1789-1798. DOI :

http://dx.doi.org/10.1145/2702123.2702327

[53] Hannah Perner-Wilson, Leah Buechley, and Mika Satomi. 2011. Handcrafting Textile Interfaces from a Kit-of-no-parts. In Proceedings of the Fifth International Conference on Tangible, Embedded, and Embodied Interaction (TEI '11). ACM, New York, NY, USA, 61-68. DOI : http://dx.doi .org/10.1145/1935701.1935715

[54] E. R. Post, M. Orth, P. R. Russo, and N. Gershenfeld. 2000. E-broidery: Design and fabrication of textile-based computing. IBM Systems Journal 39, 3.4 (2000), 840-860. DOI : http://dx.doi.org/10.1147/sj.393.0840

[55] Ivan Poupyrev, Nan-Wei Gong, Shiho Fukuhara, Mustafa Emre Karagozler, Carsten Schwesig, and Karen E. Robinson. 2016. Project Jacquard: Interactive Digital Textiles at Scale. In Proceedings of the 2016 CHI Conference on Human Factors in Computing Systems (CHI '16). ACM, New York, NY, USA, 4216-4227. DOI : http://dx.doi.org/10.1145/2858036.2858176

[56] P. Roberts, D. D. Damian, W. Shan, T. Lu, and C. Majidi. 2013. Soft-matter capacitive sensor for measuring shear and pressure deformation. In 2013 IEEE International Conference on Robotics and Automation. 3529-3534. DOI : http://dx . doi .org/10.1109/ICRA. 2013.6631071

[57] Samsung. 2019. Galaxy S10 5G. (2019). https://www . samsung. com/us/mobile/galaxy-s10-5g/

[58] Mika Satomi and Hannah Perner-Wilson. 2019. Kobakant DIY Wearable Technology Documentation. (2019). https://www. kobakant.at/DIY/ 
[59] Shieldex. 2019. Zell - RS. (2019). https://www.shieldextrading.net

[60] SKULLPAPER. 2019. Transfer Paper For Bright Fabrics. (2019). https://www. skullpaper.com/products/ transferfolie-fur-helle-stoffe-inkjet-a4

[61] Paul Strohmeier, Victor Håkansson, Cedric Honnet, Daniel Ashbrook, and Kasper Hornbæk. 2019. Optimizing Pressure Matrices: Interdigitation and Interpolation Methods for Continuous Position Input. In Proceedings of the Thirteenth International Conference on Tangible, Embedded, and Embodied Interaction (TEI '19). ACM, New York, NY, USA, 117-126. DOI : http://dx.doi.org/10.1145/3294109. 3295638

[62] Paul Strohmeier, Jarrod Knibbe, Sebastian Boring, and Kasper Hornbæk. 2018. zPatch: Hybrid Resistive/Capacitive eTextile Input. In Proceedings of the Twelfth International Conference on Tangible, Embedded, and Embodied Interaction (TEI '18). ACM, New York, NY, USA, 188-198. DOI : http://dx.doi.org/10.1145/3173225. 3173242

[63] Paul Strohmeier, Roel Vertegaal, and Audrey Girouard. 2012. With a Flick of the Wrist: Stretch Sensors As Lightweight Input for Mobile Devices. In Proceedings of the Sixth International Conference on Tangible, Embedded and Embodied Interaction (TEI '12). ACM, New York, NY, USA, 307-308. DOI : http://dx.doi.org/10.1145/2148131.2148195

[64] Seeed Studio. 2019. Seeeduino FILM. (2019). https: //www. seeedstudio. com/Seeeduino-Film-p-689.html

[65] Danmei Sun, Meixuan Chen, Symon Podilchak, Apostolos Georgiadis, Qassim S Abdullahi, Rahil Joshi,
Sohail Yasin, Jean Rooney, and John Rooney. 2019. Investigating flexible textile-based coils for wireless charging wearable electronics. Journal of Industrial Textiles (2019), 1528083719831086. DOI: http://dx.doi.org/10.1177/1528083719831086

[66] AMOHR Technische Textilien GmbH. 2019. Conductive Tapes and Textile Sensors. (2019). https://www . amohr. com

[67] Tiertime. 2019. UP Fila ABS Filament. (2019). https://www. tiertime.com/tiertime-up-fila/

[68] Vlieseline. 2019. Fusible Interlining Product Series Vliesofix; SKU: 4008983113768. (2019). https: //www.vlieseline.com/Products/Fusible-Interlinings/

[69] WEARIC. 2019. Smart Textiles. (2019). https://www. wearic.com

[70] Karl D.D. Willis, Cheng Xu, Kuan-Ju Wu, Golan Levin, and Mark D. Gross. 2011. Interactive Fabrication: New Interfaces for Digital Fabrication. In Proceedings of the Fifth International Conference on Tangible, Embedded, and Embodied Interaction (TEI '11). ACM, New York, NY, USA, 69-72. DOI : http://dx.doi.org/10.1145/1935701.1935716

[71] Raphael Wimmer and Patrick Baudisch. 2011. Modular and Deformable Touch-sensitive Surfaces Based on Time Domain Reflectometry. In Proceedings of the 24th Annual ACM Symposium on User Interface Software and Technology (UIST'11). ACM, New York, NY, USA, 517-526. DOI :

http://dx.doi.org/10.1145/2047196.2047264 